\title{
RESILIENCIA, FUNCIONAMIENTO FAMILIAR, INTELIGENCIA SOCIOEMOCIONAL EN MUJERES MALTRATADAS POR SU PAREJA Y QUE ASISTEN A UN CENTRO DE EMERGENCIA MUJER DE SURCO
}

\author{
Nelly UGARRIZA CHÁVEZ \\ Universidad Ricardo Palma \\ nugarriza@urp.edu.pe \\ Luis ESCURRA MAYAUTE \\ Universidad Nacional Mayor de San Marcos \\ lescurram@unmsm.edu.pe
}

\section{RESUMEN}

El objetivo central del estudio fue examinar las relaciones entre resiliencia, funcionamiento familiar e inteligencia emocional en mujeres maltratadas por su pareja o ex pareja. El tipo de investigación fue básica y el diseño descriptivo y correlacional, el muestreo fue no probabilístico por conveniencia, seleccionándose una muestra de 112 mujeres maltratadas por su pareja o ex pareja que asistieron al Centro de Emergencia Mujer, a las comisarías del distrito de Surco y al Consultorio Jurídico de la Universidad Ricardo Palma. Se administraron los instrumentos: la Escala de resiliencia de Wagnild y Young, el inventario de Inteligencia Emocional de BarOn y el cuestionario de Funcionamiento familiar integral de Epstein, Baldwin, y Bishop, cuya validez y confiabilidad fue demostrada. Los hallazgos más importantes establecen correlaciones positivas entre la inteligencia emocional y la resiliencia corroborándose la hipótesis 1 del presente estudio en el sentido de que las mujeres que han sido objeto de maltrato por su pareja o expareja revelan que poseen suficiente grado de inteligencia emocional, lo cual les ayuda a ser resiliente, enfrentando la adversidad a los vejámenes físicos y psicológicos al que se encuentran expuestas, y a las condiciones del contexto desfavorable en las que viven.

\section{PALABRAS CLAVES}

Resiliencia, Funcionamiento Familiar Integral, Inteligencia socioemocional, Mujeres maltratadas por su pareja o expareja.

\section{RESILIENCE, FAMILY FUNCTIONING, SOCIO-EMOTIONAL INTELLIGENCE IN WOMEN ABUSED BY THEIR PARTNER AND WHO ATTEND A SURCO WOMAN EMERGENCY CENTE}

\section{SUMMARY}

The main objective of the study was to examine the relationships between resilience, family functioning and emotional intelligence in women abused by their partner or ex-partner. The type of research was basic and the design was descriptive and 
correlational, the sampling was not probabilistic for convenience, selecting a sample of 112 women abused by their partner or couple who attended the Emergency Center Woman, the police stations of the district of Surco and the Legal Office of the Ricardo Palma University. The instruments were administered: the Wagnild and Young Resilience Scale, the Bar Emotional Intelligence Inventory and the Family Familiar Questionnaire, de Epstein, Baldwin, and Bishop, whose validity and reliability was demonstrated. The most important findings establish positive correlations between emotional intelligence and resilience, corroborating hypothesis 1 of the present study in the sense that women who have been mistreated by their partner or expartner reveal that they possess a sufficient degree of emotional intelligence, which helps them to be resilient, facing adversity to physical and psychological harassment to which they are exposed, and the conditions of the unfavorable context in which they live.

\section{KEYWORDS}

Resilience, Integral Family Functioning, Social Emotional Intelligence, Women abused by their partner or ex-partner.

Recibido: 20/04/2020

Aprobado: $24 / 07 / 2020$

\section{INTRODUCCIÓN}

a violencia en la pareja es una compleja realidad social entendida como una relación de abuso dentro de ella vivan juntos o no, en un marco formal o informal (la relación conyugal, relaciones de convivencia, relaciones de noviazgo y las relaciones de la ex-pareja). La violencia en la pareja afecta principalmente a las mujeres, el 75\% de los casos, (Velásquez, 2003; Martínez; Walter y col. 1997). Nace de la violencia de género como una forma de dominación y control social, que se presenta en todas partes, afectando a las mujeres de todos los estratos sociales, sobre todo, porque es un comportamiento aprendido como parte del proceso de socialización. Lo que se legitima con el sexismo y la misoginia, logrando que el hombre se vea limitado para el aprendizaje de la compasión y la empatía, y en las mujeres aparezcan actitudes de sumisión, dependencia y debilidad (Alberdi y Matas, 2002).

El informe publicado por la Organización Mundial de la Salud señala que la violencia de pareja es el tipo más común de violencia contra la mujer, ya que afecta al $35 \%$ de las mujeres en todo el mundo y un $38 \%$ de los feminicidios que se producen en la sociedad son cometidos por su pareja, una cifra sin duda muy preocupante (OMS, 2013). En el Perú las cifras son alarmantes ya que, en los tres últimos meses del año 2019 se han registrado $86.4 \%$ de casos de violencia contra la mujer, violencia familiar y sexual atendidos por los Centros de Emergencia Mujer - CEM - MIMP y en el mes de enero, el 32\% de los casos corresponde al departamento de Lima. En Santiago de Surco, Gonzales (2017) señala que la violencia física es uno de los tipos 
de violencia con el mayor índice de incidencia en este distrito (56\%), mientras que el de menor valor es la violencia psicológica (37\%).

Según la Ley № 30364, “Ley para prevenir, sancionar y erradicar la violencia contra las mujeres y los integrantes del grupo familiar" (El Peruano, 2015), se distinguen los siguientes tipos de violencia contra las mujeres y los integrantes del grupo familiar: "a) violencia física, b) Violencia psicológica. c) Violencia sexual. y d) Violencia económica o patrimonial.

Mientras que el maltrato físico y sexual en las relaciones interpersonales parece fácilmente identificable por los efectos observables en la víctima, en cambio, las expresiones de maltrato psicológico parecen no ser tomados en cuenta para su detección, agravándose esta situación debido a que, con frecuencia, la mujer no lo identifica como tal, sino más bien como comportamientos que demuestran el carácter propio del agresor y del amor que le tiene (Blázquez, Moreno y GarcíaBaamonde, 2009). El maltrato psicológico puede ser simultáneo a la violencia física, anteceder a la misma, o bien se puede dar con independencia de estas agresiones, es un tipo de violencia "invisible" e insidiosa que puede causar alteraciones psicosomáticas, trastornos psicológicos, agravar enfermedades físicas o, incluso, inducir al suicidio. Por otro lado, la violencia psicológica, no es reconocida como tal por la víctima ni tampoco por el contexto que la rodea, no solo por la falta de evidencia externa de sus secuelas, sino también por la tolerancia que aún existe socialmente ante estas formas de maltrato, así como la persistencia en la cultura de ciertos clichés del "amor romántico" que, en determinados sectores de la población, las conductas controladoras y posesivas se identifican con el amor (Murphy y Hoover, 1999; Blázquez, Moreno y GarcíaBaamonde, 2010).

Es factible percibir que cada conducta de maltrato de parte del agresor, señalado por Murphy y Hoover (1999), influye de manera adversa en las reacciones emocionales y sociales de la víctima destacando el miedo o sumisión, el aislamiento, dependencia, ansiedad e inseguridad en su interacción conyugal y merma de su autoestima. Cabe acotar que estas manifestaciones se pueden convertir en un patrón de conducta que puede pasar de una generación a otra, pero también existen otras personas que siendo objeto de maltrato por parte de su pareja reaccionan ante la adversidad de manera positiva, afrontando los problemas surgidos a nivel familiar de forma creativa y observando las adversidades como una experiencia de aprendizaje y de inspiración para otras personas, es decir algunas mujeres maltratadas por su pareja, muestran resiliencia, ya que la característica principal de esta conducta es la capacidad de superar la adversidad sin sucumbir a las agresiones, mostrando una actitud positiva y de superación ante la vida. Estas apreciaciones son corroboradas por algunos estudios realizados a nivel de Latinoamérica, por Lam, Contreras, Cuesta, Mori, Cordori y Carrillo (2008); Cordero y Teyes (2016), Carolin (2014) y Roa, Estrada y Tobo (2012). Cabe aclarar que, si bien es cierto que para poder hablar de resiliencia debe de existir una vivencia traumática, esto no quiere decir que el incremento de violencia física o psicológica traiga consigo un aumento en el nivel de resiliencia, aunque la estadística corresponde a esta lógica. 


\section{ANTECEDENTES CONCEPTUALES}

La resiliencia en Psicología aún se encuentra en proceso de desarrollo siendo considerado como un concepto contemporáneo el cual ha alcanzado fuerza en las últimas dos décadas, y posee un carácter heterogéneo el cual varía de acuerdo al marco teórico que se adopte como referente. La literatura científica demuestra que la resiliencia es una respuesta común y su aparición no indica patología, sino un ajuste saludable a la adversidad, esta se construye desde las fortalezas y potencialidades del individuo, tomando en cuenta la etapa del desarrollo en que se encuentre, siendo un proceso dinámico, donde la relación con el ambiente son condiciones necesarias para su desenvolvimiento, es decir, desde la adversidad la resiliencia cobra significado. A partir de las diferentes maneras de reacción ante circunstancias percibidas como adversas, mientras algunas personas sucumben, otras por el contrario se desarrollan exitosamente a pesar de la adversidad, convirtiendo a esta, en un trampolín para el crecimiento y desarrollo de la persona (Cordero y Teyes, 2016).

En este trabajo se ha adoptado un enfoque basado en el modelo ecológico "descentralizado" de la resiliencia de Ungar (2011), quien da significado a las fuerzas que emergen tanto de la persona como del contexto, por lo que se define la resiliencia como la capacidad del individuo para navegar de manera exitosa en busca de los recursos que sustentan su bienestar y la negociación con aquellos que controlan y proveen los recursos, asegurando que sean significativos culturalmente para aquellos que requieren apoyo. Esta definición no solamente enfatiza la capacidad de las personas para moverse en ambientes peligrosos, sino también la capacidad del ambiente de habilitar los recursos que las mujeres que son maltratadas necesitan en forma apropiada. Es decir, la resiliencia surge cuando el individuo es capaz de hacer uso de factores internos (por ejemplo, inteligencia emocional, afrontar retos, revelar autoeficacia, autoimagen positiva, aptitudes físicas e intelectuales, competencias de planificación, entre otras características personales) y recursos externos (por ejemplo, satisfacción de sus necesidades físicas, contar con apoyos familiares y sociales saludables, entre otros). Estos recursos externos deben ser accesibles, significativos y relevantes según la cultura en que se desarrollan las personas. Además, esta definición enfatiza que las personas tienen que ser apoyadas para encontrar los recursos, lo que depende en buena medida de su disponibilidad y accesibilidad; y que tienen que ser guiadas hacia los recursos apropiados, es decir, significativos en el contexto cultural de la comunidad al que pertenecen y donde se expresan (Ungar, 2010).

Por otro lado, Fergus y Zimmerman (2005) expresan que la resiliencia, involucra un proceso para superar los efectos negativos de la exposición al riesgo, afrontar exitosamente las experiencias traumáticas y evitar las circunstancias negativas asociadas con el riesgo. Para que se desarrolle la resiliencia tienen que estar presentes tanto factores de protección como de riesgo. Los factores protectores son concebidos como aquellas condiciones o entornos capaces de favorecer el desarrollo de las personas y de reducir los efectos negativos de circunstancias adversas. En cambio, los factores de riesgo son aquellas condiciones personales, 
socioeconómicas, familiares, biológicas o contextuales adversas que están asociadas $u$ originan conductas problemas o una salud deficiente. Las personas resilientes no sólo son capaces de enfrentar los factores estresantes y la adversidad, sino que además el ser resiliente les ayuda a disminuir la intensidad del estrés, la ansiedad, la depresión y les permite aumentar la curiosidad y su salud mental, asimismo el incremento de los factores de protección reduce el riesgo de la persona de presentar conductas problema.

Para Wagnild y Young (1993), la resiliencia es una característica de la personalidad que modera el efecto negativo del estrés y fomenta la adaptación. Ello connota vigor y fibra emocional y se ha utilizado para describir a personas que muestran valentía y adaptabilidad ante infortunios de la vida.

En cuanto al funcionamiento familiar, se puede sostener que ninguna familia se ve exenta de crisis o dificultades que pueden impedir su desarrollo a lo largo de su ciclo vital, pero al mismo tiempo cuenta con recursos que pueden ayudarle a afrontar y superar las adversidades, por lo que se puede afirmar que existe también una "resiliencia familiar". Esta expresión designa los procesos de superación y adaptación que tiene lugar en este sistema, como unidad funcional. La perspectiva sistémica permite comprender los procesos familiares que moderan el estrés y posibilitan a las familias afrontar penurias prolongadas y dejar atrás situaciones de crisis, como es el caso de ser agraviado por su pareja o expareja. Asi, Walsh (2004) refiere que el modo en como el sistema familiar enfrente y mantenga la experiencia disociadora, disminuya el estrés, se reorganice con eficacia y siga adelante con su vida, influirá en la adaptación inmediata y mediata de todos sus integrantes, así como en la supervivencia y bienestar de la unidad familiar.

Existen diversos modelos explicativos sobre el funcionamiento familiar y para este estudio se ha considerado en modelo multidimensional de McMaster (MMFF) (Epstein, Bishop y Levine, 1978), derivado de la experiencia clínica y que se basa en la teoría sistémica describiendo la estructura, organización y patrones de interacciones de la unidad familiar y para explicarlo proponen evaluar las siguientes dimensiones: Resolución de problema, comunicación, roles, capacidad de respuestas afectivas, involucramiento afectivo, control de la conducta y el dominio de funcionamiento familiar general, que brinda una imagen integral del funcionamiento de la familia en múltiples áreas.

En relación a la Inteligencia Emocional se ha adoptado el Modelo Multifactorial de Bar-On (1997a), quien define a la inteligencia emocional como un conjunto de habilidades personales, emocionales y sociales y de destrezas que influyen en nuestra habilidad para adaptarse y enfrentar a las demandas y presiones del medio. Como tal, nuestra inteligencia no cognitiva es un factor importante en la determinación de la habilidad para tener éxito en la vida, influyendo directamente en el bienestar general y en la salud emocional. La estructura del modelo de BarOn de inteligencia no cognitiva puede ser vista desde un enfoque sistémico, que considera cinco componentes mayores de la inteligencia emocional y sus respectivos subcomponentes que se aprecian en la Tabla 1. 
Tabla 1. Enfoque Sistémico de los 15 Subcomponentes de Inteligencia Emocional y Social del I-CE de BarOn

\begin{tabular}{l|l}
\hline COMPONENTES & \multicolumn{1}{c}{ SUBCOMPONENTES } \\
\hline & $\begin{array}{l}\text { Comprensión emocional de sí mismo (CM): la habilidad } \\
\text { para percatarse y comprender nuestros sentimientos y } \\
\text { emociones, diferen ciarlos y conocer el porqué de los } \\
\text { mismos. } \\
\text { Asertividad (AS): La habilidad para expresar sentimientos, } \\
\text { creencias y pensamientos sin dañar los sentimientos de los } \\
\text { demás; y defender nuestros derechos de una manera no } \\
\text { destructiva. } \\
\text { Autoconcepto (AC): La habilidad para comprender, aceptar } \\
\text { y respetarse a sí mismo, aceptando nuestros aspectos } \\
\text { positivos y negativos, como también nuestras limitaciones } \\
\text { y posibilidades. } \\
\text { Autorrealización (AR): La habilidad para realizar lo que } \\
\text { realmente podemos, queremos y disfrutamos de hacerlo. } \\
\text { Independencia (IN): Es la habilidad para autodirigirse, } \\
\text { sentirse seguro de sí mismo en nuestros pensamientos, } \\
\text { acciones y ser independientes emocionalmente para tomar } \\
\text { nuestras decisiones. }\end{array}$ \\
\hline
\end{tabular}

Empatía (EM): La habilidad de percatarse, comprender, y apreciar los sentimientos de los demás.

Relaciones interpersonales (RI): La habilidad para establecer y mantener relaciones mutuas satisfactorias que son caracterizadas por una cercanía emocional e intimidad. Responsabilidad social (RS): La habilidad para demostrarse a sí mismo como una persona que coopera, contribuye y que es un miembro constructivo del grupo social.

Solución de problemas (SP): La habilidad para identificar y definir los problemas como también para generar e implementar soluciones efectivas.

Prueba de la realidad (PR): La habilidad para evaluar la correspondencia entre lo que experimentamos (lo subjetivo) y lo que en la realidad existe ( lo objetivo).

Flexibilidad (FL): La habilidad para realizar un ajuste adecuado de nuestras emociones, pensamientos y conductas a situaciones y condiciones cambiantes.

Tolerancia al estrés (TE): La habilidad para soportar eventos adversos, situaciones estresantes, y fuertes emociones sin "desmoronarse", enfrentando activa y positivamente el estrés.

Control de los impulsos (CI): La habilidad para resistir o postergar un impulso o tentaciones para actuar y controlar nuestras emociones. 
Felicidad (FE): La habilidad para sentirse satisfecho con nuestra vida, para disfrutar de sí mismo y de otros y para divertirse y expresar sentimientos positivos.

Optimismo (OP): La habilidad para ver el aspecto más brillante de la vida y mantener una actitud positiva a pesar de la adversidad y los sentimientos negativos.

Nota: Adaptado de Bar-On, R. (1997a)

El modelo de inteligencia del Bar-On (1997a), sostiene que las personas saludables que funcionan bien y son exitosas poseen un grado suficiente de inteligencia emocional que se combina con otros determinantes importantes de nuestra habilidad para tener éxito en adaptarse a las demandas del medio ambiente, tales como las características básicas de la personalidad y la capacidad intelectual cognitiva. Las personas emocionalmente inteligentes son capaces de reconocer y expresar sus emociones, comprenderse a sí mismos, actualizar sus capacidades potenciales, llevar una vida regularmente saludable y feliz. Son capaces de entender la manera como las otras personas se sienten, de tener y mantener relaciones interpersonales satisfactorias y responsables, sin llegar a ser dependientes de los demás. Son generalmente optimistas, flexibles, realistas, tienen éxito en resolver sus problemas y afrontar el estrés, sin perder el control.

Se ha podido apreciar que son escasos los estudios que relacionen inteligencia emocional y la resiliencia, Almeida, Omar, Aguiar, \& Carvalho, (2009) señalan que existe asociación significativa entre ambos constructos, lo que estaría indicando que los jóvenes más inteligentes emocionalmente tendrían la habilidad para transformar las emociones positivas en oportunidades de aprendizaje y las emociones negativas en crecimiento positivo frente a las adversidades. De allí que los objetivos de este estudio sea analizar las relaciones entre la resiliencia, el funcionamiento familiar y la inteligencia socioemocional, en mujeres que son víctimas del maltrato físico y/o psicológico por su pareja o expareja y que asisten a un Centro de Emergencia Mujer de Surco y establecer las diferencias con respecto a las variables mencionadas considerando la edad y el estado conyugal de las mujeres maltratada por su pareja.

\section{HIPÓTESIS}

$\mathrm{H}_{1}$ : Existe relación entre la resiliencia, el funcionamiento familiar y la inteligencia socioemocional en mujeres que son víctimas del maltrato por su pareja y que asisten a un Centro de Emergencia Mujer de Surco.

$\mathrm{H}_{2}$ : Existe diferencias significativas con respecto a las variables mencionadas en mujeres que son víctimas del maltrato por su pareja considerando la edad y el estado conyugal de las agraviadas. 


\section{MÉTODO}

Esta investigación es de carácter descriptivo y el diseño es transeccional correlacional siendo su finalidad establecer relaciones entre las variables resiliencia, funcionamiento familiar inteligencia emocional en mujeres maltratadas por su pareja o expareja tal como se dan en el presente y comparar las diferencias considerando la edad y el estado conyugal de las agraviadas. (Hernández, Fernández, y Baptista, 2014).

\section{Muestra}

El muestreo fue no probabilístico por conveniencia seleccionándose mujeres denunciantes adultas que han sido objeto de maltrato físico y/o psicológico o de violencia sexual por parte de su pareja o expareja, en un periodo comprendido entre setiembre a diciembre del 2018.

Se estableció un tamaño mínimo de 112 mujeres agraviadas obtenido al aplicar el programa estadístico G*Power (Faul, Erdfelder, Buchner y Lang, 2009, 2014). Ellas residen en el distrito de Santiago de Surco, el $57.1 \%$ se ubica en un rango de edad de 41 a 61 años, el mayor nivel educativo alcanzado corresponde a secundaria en un $54.5 \%$ y en cuanto a su estado conyugal el $56.3 \%$ viven con su pareja y el $43.7 \%$ se encuentra sin pareja.

\section{Instrumentos}

La información fue recabada mediante los siguientes instrumentos:

1. Una ficha de información demográfica de la familia para registrar la edad, el estado civil y el mayor nivel educativo alcanzado por la madre.

2. La escala de resiliencia de Wagnild y Young (1993). Se utilizó la adaptación cultural de la Escala de Resiliencia realizada en Argentina, Bolivia, República Dominicana y Perú (Salgado, 2012). Se realizaron ligeros cambios en la redacción de los ítems para facilitar su comprensión, se omitieron los ejemplos en las instrucciones y las alternativas de respuestas se expresaron en un rango de 1 a 5 . Todos los ítems cuentan con una redacción positiva, por lo que un puntaje alto indica una mayor resiliencia. Los factores y componentes se presentan en la Tabla 2.

Tabla 2. Factores y componentes de la escala de resiliencia

\begin{tabular}{ll}
\hline \multicolumn{1}{c}{ FACTORES } & \multicolumn{1}{c}{ COMPONENTES } \\
\hline \multirow{2}{*}{ Competencia Persona(FCP) } & Ecuanimidad (E) \\
& Satisfacción personal (SP) \\
\cline { 2 - 2 } Factor aceptación de uno mismo y de la vida & Sentirse bien solo (SBS) \\
(AUV) & Confianza en sí mismo (CSM) \\
\hline
\end{tabular}

Nota: Adaptado de Wagnild, G. y Young. H. (1993) 
Las evidencias de validez de contenido, mediante la correlación entre el puntaje total de la Escala de Resiliencia y sus respectivos componentes se observan en la Tabla 3, apreciándose efectos elevados.

Tabla 3. Correlaciones Momento Producto de Pearson entre el puntaje total de la Escala de Resiliencia y los componentes

\begin{tabular}{lccc}
\hline \multicolumn{1}{c}{ Resiliencia } & 1 & 2 & 3 \\
\hline 1. Competencia Personal & - &, $75^{* *}$ &, $99^{* *}$ \\
2. Aceptación de uno mismo y de la vida & & - &, $85^{* *}$ \\
3. Puntaje total en Resiliencia & & - \\
\hline
\end{tabular}

$* * p<.01$

Con respecto a las evidencias de confiabilidad el coeficiente Alpha de Cronbach fue .89 y el Omega de McDonald .90, considerándose altos.

Tabla 4. Correlaciones Alfa de Cronbach para la escala de resiliencia

Alfa de Cronbach Omega de McDonald N de elementos

\begin{tabular}{lll}
\hline 0,890 & 0,90 & 20 \\
\hline
\end{tabular}

3. Evaluación del funcionamiento familiar integral. Esta escala conforma uno de las siete dimensiones diseñadas para evaluar a las familias de acuerdo con el Modelo McMaster de Funcionamiento Familiar (Epstein, Baldwin, y Bishop, 1978). En este estudio las evidencias de confiabilidad para la escala de funcionamiento familiar integral arrojaron coeficientes similares para el Alfa de Cronbah ( $a=.862)$ y el Omega de Mac Donald $(\omega=, 864)$ (Tabla 5).

Tabla 5. Correlaciones Alfa de Cronbach para la escala de funcionamiento familiar integral

\begin{tabular}{ccc}
\hline Alfa de Cronbach & Omega de McDonald & N de elementos \\
\hline 0,862 & 0,864 & 12 \\
\hline
\end{tabular}

Respecto a la confiabilidad tanto Alfa de Cronbach, como Omega de Mac Donald, si el elemento se suprimía fue elevada oscilando entre .85 y .86, por lo que no se eliminó ningún ítem.

Con respecto a la evidencia de consistencia interna de la escala se demostró mediante la correlación Ítems- Puntaje Total, y los resultados oscilan entre .53 y .72. Los valores se presentan en la Tabla 6. 
Tabla 6. Correlaciones Alfa de Cronbach para los Ítems de la escala de funcionamiento familiar integral y Correlación de Pearson Ítems- Puntaje Total

\begin{tabular}{|c|c|c|c|}
\hline Ítems de la escala & $\begin{array}{c}\text { a si el } \\
\text { elemento } \\
\text { se ha } \\
\text { suprimido }\end{array}$ & $\begin{array}{l}\omega \text { si el } \\
\text { elemento } \\
\text { se ha } \\
\text { suprimido }\end{array}$ & $\begin{array}{l}\text { Rho } \\
\text { Ítems- } \\
\text { Puntaje } \\
\text { Total }\end{array}$ \\
\hline $\begin{array}{l}1 \text { Planear actividades familiares es difícil porque no } \\
\text { nos comprendemos. }\end{array}$ & .86 & .86 & $.72^{* * *}$ \\
\hline 2 En tiempos difíciles buscamos el apoyo mutuo. & .86 & .86 & $.53^{* *}$ \\
\hline $\begin{array}{l}3 \text { No podemos hablar entre nosotros de la tristeza } \\
\text { que sentimos. }\end{array}$ & .86 & .86 & $.55^{* *}$ \\
\hline 4 Cada uno es aceptado por lo que es. & .85 & .85 & $.61^{* *}$ \\
\hline $\begin{array}{l}5 \text { Evitamos hablar de nuestros miedos y } \\
\text { preocupaciones. }\end{array}$ & .85 & .85 & $.66^{* *}$ \\
\hline $\begin{array}{l}6 \text { Podemos expresarnos nuestros sentimientos } \\
\text { mutuamente. }\end{array}$ & .85 & .85 & $.68^{* *}$ \\
\hline $\begin{array}{l}7 \text { Existen muchos malos sentimientos en nuestra } \\
\text { familia. }\end{array}$ & .85 & .85 & $.65^{* *}$ \\
\hline $\begin{array}{l}8 \text { Sentimos que somos aceptados por lo que } \\
\text { somos. }\end{array}$ & .85 & .85 & $.56^{* *}$ \\
\hline $\begin{array}{l}9 \text { Tomar decisiones es algo difícil para nuestra } \\
\text { familia. }\end{array}$ & .86 & .86 & $.56^{* *}$ \\
\hline $\begin{array}{l}10 \text { Podemos tomar decisiones para resolver } \\
\text { problemas. }\end{array}$ & .85 & .85 & $.60^{* *}$ \\
\hline 11 No nos llevamos bien. & .85 & .85 & $.71 * *$ \\
\hline 12 Nos hacemos confidencias mutuamente. & .85 & .85 & $.65^{* *}$ \\
\hline
\end{tabular}

${ }^{* *} p<.01$

4. El Inventario del Cociente Emocional para jóvenes y adultos (ICE-JA), Baron (1997a), adaptado en Lima-Perú por Ugarriza (2001) en una muestra representativa de 1996 sujetos de Lima Metropolitana, varones y mujeres, de 16 años y más. El análisis factorial confirmatorio de segundo orden sobre los componentes del ICE ha verificado la estructura factorial 5-1 propuesta por el modelo multifactorial de la inteligencia emocional de Bar-On (1997a). Los cinco componentes son: intrapersonal $(\mathrm{CIA})$, interpersonal $(\mathrm{CIE})$, adaptabilidad (CAD), manejo de estrés (CME) y estado de ánimo en general (EAM). Los 15 subcomponentes de Inteligencia Emocional y Social del I-CE de BarOn se han presentado en la Tabla 1.

Para el presente estudio se ha elaborado una forma abreviada del ICE-JA, habiéndose seleccionado 60 ítems de los 113 que integran la escala general.

Se aprecia en la Tabla 7 que el índice de confiabilidad para la variable de inteligencia emocional total fue de .91 en el Alfa y .96 en el Omega considerándose como muy altos, y para cuatro de los subcomponentes oscilan entre valores altos de .70 a .77 
y moderado .60 para el componente manejo del estrés, interpretación realizada de acuerdo al criterio de Cronbach, tal como lo cita Thorndike y Hagen (1989).

Tabla 7. Confiabilidad Alfa de Cronbach y Omega de McDonald para las escalas de inteligencia emocional administradas a mujeres maltratadas por su pareja

\begin{tabular}{lccl}
\hline \multicolumn{1}{c}{ Variables } & N de reactivos & $a$ & $\omega$ \\
\hline Inteligencia emocional & 60 & .91 & .96 \\
Intrapersonal & 20 & .75 & .76 \\
Interpersonal & 12 & .77 & .77 \\
Adaptabilidad & 12 & .70 & .70 \\
Manejo del estrés & 8 & .60 & .61 \\
Estado de ánimo general & 8 & .75 & .76 \\
\hline
\end{tabular}

En la Tabla 8 se puede apreciar evidencias de consistencia interna elevadas para la escala abreviada de inteligencia emocional. Las correlaciones son estadísticamente significativas para todos los componentes siendo el tamaño del efecto alto; excepto entre el intrapersonal con manejo del estrés, y este último con estado de ánimo general. Además el efecto es bajo entre el componente interpersonal con manejo del estrés.

Tabla 8. Correlaciones Momento Producto de Pearson entre el puntaje Total de la escala de Inteligencia emocional y los componentes

\begin{tabular}{lcccccc}
\hline \multicolumn{1}{c}{ Inteligencia emocional } & 1 & 2 & 3 & 4 & 5 & 6 \\
\hline 1. Intrapersonal & - & $.67^{* *}$ & $.61^{* *}$ & $.43^{* *}$ & $.78^{* *}$ & $.91^{* *}$ \\
2. Interpersonal & & - & $.51^{* *}$ & $.23^{*}$ & $.65^{* *}$ & $.79^{* *}$ \\
3. Adaptabilidad & & & - & $.56^{* *}$ & $.57^{* *}$ & $.80^{* *}$ \\
4. Manejo del estrés & & & & - & $.39^{* *}$ & $.60^{* *}$ \\
$\begin{array}{l}\text { 5. Estado de ánimo general } \\
\text { 6. Puntaje Total Inteligencia }\end{array}$ & & & & & - & $.85^{* *}$ \\
$\quad$ Emocional & & & & & - \\
\hline
\end{tabular}

${ }^{*} \mathrm{p}<.05$

$* * p<.01$

\section{Procedimientos}

Se contó con la autorización del Ministerio de la Mujer y Poblaciones vulnerables y la del Vicerrectorado Académico de la Universidad Ricardo Palma. La recolección de datos fue realizada por dos psicólogos con experiencia con poblaciones vulnerables. Se aplicaron las encuestas individualmente, previa entrevista y consentimiento informado de la persona agraviada, dejando claro que los resultados serían publicados pero su nombre mantenido en el anonimato. Se contó con los permisos correspondientes de las personas que han adaptado los instrumentos en la realidad peruana. La aplicación de encuestas se realizó entre los meses de setiembre a diciembre y luego se procedió a desarrollar la base de datos y el análisis estadístico correspondiente utilizando el SPSS versión 25. 


\section{RESULTADOS}

\section{Análisis descriptivos}

La puntuaciones total de los instrumentos aplicados a la muestra de mujeres maltratadas por su pareja fueron sometidas a la prueba de bondad de ajuste de Smirnov - Kolgomorov, se hallaron valores K-S significativos, en nueve de diez variables, lo que significa que la mayor parte de las distribuciones de los puntajes analizados se aproximan a la curva de distribución normal, razón por la cual se emplearon estadísticos paramétricos en el análisis de los datos. (Siegel \& Castellan, 1995), excepto en el factor I de competencia personal de la Escala de Resiliencia, cuyo puntaje no se ajustaba a una distribución normal.

\section{Relaciones entre la inteligencia emocional, la resiliencia y el funcionamiento Familiar Integral}

En la tabla 9 se puede apreciar los resultados de las correlaciones de las variables de las escalas administradas a las mujeres maltratadas por su pareja. El examen reveló correlaciones positivas significativas $p<.01$ entre la inteligencia emocional total y la puntación total de la escala de resiliencia $r=.56$, con el factor aceptación de uno mismo y de la vida $r=.54$, con el factor competencia personal $r=.53$. Asimismo, fue factible observar correlaciones positivas significativas al uno por ciento entre cada uno de los componentes de inteligencia emocional con cada uno de los factores que evalúa la escala de resiliencia.

Las correlaciones entre inteligencia emocional total y funcionamiento familiar integral son significativas y negativas $p<.01, r=-.59$, lo mismo ocurre en las correlaciones entre los componentes de la escala de inteligencia emocional y el funcionamiento familiar que se dan entre -.33 y -.56, siendo el tamaño del efecto grande, lo mismo ocurre con los componentes intrapersonal, interpersonal y estado de ánimo general. En cambio con adaptabilidad, manejo del estrés, resiliencia y sus factores competencia personal y aceptación de uno mismo y de la vida el tamaño del efecto es moderado. En resumen, en el resto de las correlaciones entre las diversas variables el efecto es alto, pues las correlaciones son significativas sobrepasando $r=.50$. Las correlaciones entre .34 y .49 son significativas y de efecto moderado. La correlación entre adaptabilidad y el factor de competencia personal de resiliencia ( $r=.28$ ) es significativo, pero el tamaño del efecto es bajo.

Tabla 9. Correlaciones de Pearson entre las escalas de inteligencia emocional, resiliencia y funcionamiento familiar integral, administradas a las mujeres maltratadas por su pareja

\begin{tabular}{llccc}
\hline \multicolumn{1}{c}{ Variables } & 7 & 8 & 9 & 10 \\
\hline 1 Inteligencia emocional Total & $.56^{* *}$ & $.53^{* *}$ & $.54^{* *}$ & $-.59^{* *}$ \\
2 Intrapersonal & $.51^{* *}$ & $.50^{* *}$ & $.48^{* *}$ & $-.56^{* *}$ \\
3 Interpersonal & $.44^{* *}$ & $.45^{* *}$ & $.41^{* *}$ & $-.50^{* *}$ \\
4 Adaptabilidad & $.38^{* *}$ & $.28^{* *}$ & $.39^{* *}$ & $-.37^{* *}$ \\
5 Manejo del estrés & $.37^{* *}$ & $.34^{* *}$ & $.36^{* *}$ & $-.33^{* *}$ \\
6 Estado de ánimo general & $.53^{* *}$ & $.55^{* *}$ & $.49^{* *}$ & $-.55^{* *}$ \\
\hline & & & & \\
\hline
\end{tabular}


Continúa Tabla 9

\begin{tabular}{llcc}
7 Puntaje total de resiliencia (PTR) & - & - & $-.45^{* *}$ \\
8 Competencia personal (CP) & - & - & $-.46^{* *}$ \\
9 Aceptación de uno mismo y de la vida (AUV) & - & - & $-.42^{* *}$ \\
10 Funcionamiento Familiar integral (FFI) & - & - & - \\
\hline
\end{tabular}

${ }^{* *} \mathrm{p}<.01$

En la Tabla 10 se presentan los resultados de los cuestionarios según el grupo etario de las mujeres objeto de maltrato por su pareja o expareja, existen diferencias significativas en el componente de adaptabilidad de inteligencia emocional. En adaptabilidad alcanzó una media de 43.00 para el grupo etario de 41 a 60 años y de 39.78 para el de 19 a 40 años, siendo el test t de Student de -2.54 y la diferencia estadísticamente significativa $\mathrm{p}<.05$, apreciándose además un tamaño del efecto mediano de .50 según la d de Cohen $(1988,1992)$. En el factor de competencia personal de la escala de resiliencia, la media para el grupo de mayor edad fue de 67.88 y para el de menor edad de 64.66 , el test t de Student fue de -266, siendo la diferencia significativa a un nivel de $p<.05$ y la d de Cohen .38 denota un tamaño del efecto mediano. El mismo efecto se observa en las comparaciones según la edad, aunque las diferencias no son significativas, en las variables de inteligencia emocional, intrapersonal, resiliencia y el factor competencia personal.

Tabla 10. Comparación de las puntuaciones obtenidas con el test t de Student de las escalas de inteligencia emocional, resiliencia y funcionamiento familiar integral, administradas a las mujeres maltratadas según la edad ( $N=112)$

\begin{tabular}{|c|c|c|c|c|c|}
\hline \multirow{3}{*}{ Variables } & \multicolumn{2}{|c|}{ Edad (años) } & \multirow{3}{*}{$t$} & \multirow{3}{*}{$p$} & \multirow{3}{*}{$\begin{array}{l}\text { d de } \\
\text { Coher }\end{array}$} \\
\hline & $\begin{array}{l}19 \text { a } 40 \\
(n=64)\end{array}$ & $\begin{array}{l}41 \text { a } 60 \\
(n=48)\end{array}$ & & & \\
\hline & $M(D E)$ & $M(D E)$ & & & \\
\hline Inteligencia emocional & $204.78(29.46)$ & $214.73(27.46)$ & -1.82 & .069 & .35 \\
\hline Intrapersonal & $67.23(10.69)$ & $71.06(9.82)$ & -1.94 & .055 & .37 \\
\hline Interpersonal & $43.61(7.34)$ & $45.33(7.48)$ & -1.22 & .227 & .23 \\
\hline Adaptabilidad & $39.78(7.18)$ & $43.00(5.84)$ & -2.54 & .013 & .50 \\
\hline Manejo del estrés & $25.59(4.67)$ & $26.13(5.28)$ & -.56 & .581 & .11 \\
\hline Estado de ánimo general & $28.56(6.14)$ & $29.21(5.94)$ & -.56 & .576 & .11 \\
\hline Resiliencia & $81.06(11.52)$ & $84.77(8.95)$ & -1.92 & .067 & .36 \\
\hline Competencia personal & $64.66(9.37)$ & $67.88(7.12)$ & -2.66 & .033 & .38 \\
\hline $\begin{array}{l}\text { Aceptación uno mismo y de } \\
\text { la vida }\end{array}$ & $16.41(2.58)$ & $16.90(2.43)$ & -1.02 & .743 & .20 \\
\hline $\begin{array}{l}\text { Funcionamiento familiar } \\
\text { integral }\end{array}$ & $25.56(6.51)$ & $25.10(7.32)$ & .35 & .732 & .07 \\
\hline
\end{tabular}

${ }^{* *} \mathrm{p}<.05$

Al analizar los resultados con el test t de Student teniendo en cuenta el estado conyugal con pareja y sin pareja, en la tabla 11, no se observan diferencias 
estadísticamente significativas entre las diversas medias comparadas sin embargo en las variables inteligencia emocional, manejo del estrés y estado de ánimo general, el tamaño del efecto es moderado.

Tabla 11. Comparación de las puntuaciones obtenidas con el test t de Student de las escalas de inteligencia emocional, resiliencia y funcionamiento familiar integral, administradas a las mujeres maltratadas según el estado conyugal ( $N=112)$

\begin{tabular}{lccccc}
\hline & \multicolumn{2}{c}{ Estado conyugal } & $t$ & $p$ & $\begin{array}{c}\mathrm{d} \text { de } \\
\text { Cohen }\end{array}$ \\
\cline { 2 - 3 } \multicolumn{1}{c}{ Variables } & $\begin{array}{c}\text { Con pareja } \\
(\mathrm{n}=63)\end{array}$ & $\begin{array}{c}\text { Sin pareja } \\
(\mathrm{n}=49)\end{array}$ & & & \\
& $M(D E)$ & $M(D E)$ & & & \\
\cline { 2 - 4 } Inteligencia emocional & $212.86(30.91)$ & $204.14(25.65)$ & 1.59 & .114 & .31 \\
$\quad$ Intrapersonal & $69.79(11.20)$ & $67.69(9.31)$ & 1.06 & .294 & .20 \\
Interpersonal & $44.70(7.65)$ & $43.90(7.15)$ & .57 & .573 & .11 \\
$\quad$ Adaptabilidad & $41.98(6.95)$ & $40.10(6.53)$ & 1.46 & .147 & .28 \\
Manejo del estrés & $26.57(4.87)$ & $24.86(4.87)$ & 1.85 & .067 & .35 \\
$\quad$ Estado de ánimo general & $29.81(6.16)$ & $27.60(5.69)$ & 1.95 & .053 & .37 \\
Resiliencia & $83.44(10.96)$ & $81.63(10.17)$ & .741 & .461 & .17 \\
$\quad$ Competencia personal & $66.92(8.77)$ & $64.90(8.31)$ & .749 & .456 & .24 \\
$\begin{array}{l}\text { Aceptación de uno mismo y } \\
\text { de la vida }\end{array}$ & $16.52(2.66)$ & $16.73(2.34)$ & .452 & .652 & .08 \\
$\quad \begin{array}{l}\text { Funcionamiento familiar } \\
\text { integral }\end{array}$ & $24.65(7.25)$ & $26.29(6.22)$ & -1.26 & .211 & .24 \\
\hline
\end{tabular}

${ }^{* *} \mathrm{p}<.05$

\section{DISCUSIÓN}

Los hallazgos más importantes establecen correlaciones positivas entre la inteligencia emocional y la resiliencia corroborándose la hipótesis 1 del presente estudio en el sentido de que las mujeres que han sido objeto de maltrato por su pareja o expareja revelan que poseen suficiente grado de inteligencia emocional, lo cual les ayuda a ser resilientes, enfrentando la adversidad a los vejámenes físicos y psicológicos al que se encuentran expuestas, y a las condiciones del contexto desfavorable en el que viven. Ellas demostraron ser emocionalmente inteligentes en el sentido de ser capaces, de percatarse y comprender sus sentimientos y emociones.

Asimismo se hallaron correlaciones significativas entre cada uno de los componentes de inteligencia emocional con los factores de la resiliencia, lo que muestra que las personas emocionalmente inteligentes son capaces de reconocer sus emociones, ser empáticos, mantener relaciones interpersonales satisfactorias, son hábiles para identificar los problemas y ofrecer soluciones positivas, y denotan un estado de ánimo general positivo. Estos aspectos se relacionan con el factor I competencia personal de la resiliencia, reflejando independencia, confianza en sí mismo, capacidad de afronte a tiempos difíciles, perseverancia, autodisciplina, 
mantener interés por las cosas, energía para emprender acciones que tiene que cumplir, adaptación y habilidad para experimentar paz a pesar de la adversidad. Además, la inteligencia socioemocional se asocia con el factor II aceptación de uno mismo y de la vida lo cual demuestra ecuanimidad y satisfacción personal.

Las correlaciones entre la inteligencia socioemocional y el funcionamiento familiar integral son significativas lo que indicaría que existen sentimientos y relaciones positivas no con la pareja o expareja, pero posiblemente con los otros miembros que integran el grupo familiar.

Estos resultados coinciden con la posición de Almeida, Omar, Aguiar, \& Carvalho, (2009), quiénes señalan que existen asociaciones significativas entre inteligencia emocional y resiliencia, ya que los jóvenes más inteligentes emocionalmente tendrían mayor habilidad para transformar las emociones positivas en oportunidades de aprendizaje y las emociones negativas en crecimiento positivo frente a las adversidades. Esta apreciación, si bien referida a jóvenes se puede hacer extensiva a las mujeres objeto de maltrato por su pareja o ex pareja quienes hacen frente a la adversidad, pues como señala Salgado, (2005) son capaces de transformar las carencias en una óptica de fortalezas y potencialidades para enfrentar la adversidad y a partir de ella construir una postura positiva frente al sufrimiento y al dolor. Sin embargo, cabe señalar que existen diferentes modos de reaccionar ante circunstancias percibidas como adversas, ya que mientras algunas personas sucumben a dichas circunstancias, otras por el contrario se desarrollan saludablemente a pesar de la adversidad, convirtiendo la aflicción, en un trampolín para el crecimiento y desarrollo de la persona (Cordero y Teyes, 2016).

Desde 1993, Wagnild y Young conceptualizaron la resiliencia como una característica de la personalidad que modera el efecto negativo del estrés y fomenta la adaptación. Las personas resilientes muestran valentía y adaptabilidad ante los infortunios de la vida. La resiliencia es la capacidad para resistir, tolerar la presión, los obstáculos y pese a ello hacer las cosas satisfactoriamente, describe a las personas que muestran coraje y capacidad de adaptación a pesar de las desgracias de la vida. Así, Carolin (2014) en un estudio sobre los factores de resiliencia en mujeres jefas de hogar víctimas de violencia intrafamiliar, maltrato y abuso, demostró a través de las entrevistas que luchar por una mejor vida para ellas y sus hijos les permitió encontrar un verdadero significado en la vida, manifestando como propósito el compromiso de trabajar para el futuro de sus hijos y seguir superándose.

Por otro lado, al analizar las diferencias considerando el grupo etario se halló diferencia significativa en el componente de adaptabilidad de inteligencia emocional y en el factor de competencia personal de resiliencia denotando un mayor nivel de adaptación y una mayor habilidad para solucionar problemas las mujeres mayores cuyas edades estaban comprendidas entre los 40 a 61 años, corroborándose parcialmente la hipótesis 2. Estos resultados expresarían que tienen una mayor habilidad para efectuar un ajuste adecuado de sus emociones y pensamientos frente a circunstancias cambiantes, también expresan mayor capacidad para identificar situaciones conflictivas y proponer soluciones efectivas y son más hábiles en distinguir entre lo que experimentan y lo que en realidad existe. 
En las comparaciones según el estado conyugal (con pareja o sin pareja) sólo se aprecian una tendencia a un estado de ánimo general más elevado en las mujeres maltratadas con pareja, pero no llega a ser significativo.

Posiblemente una de las limitaciones de esta investigación haya sido el no considerar de manera integral la definición de Ungar (2011) sobre resiliencia, poniendo sólo énfasis en los factores personales internos y descuidar las fuerzas que emergen también de la capacidad del ambiente de habilitar los recursos necesarios que las mujeres que son maltratadas necesitan en forma apropiada. Estos medios externos deben ser accesibles, significativos y relevantes según el contexto cultural en que se desarrollan las personas.

\section{CONCLUSIONES}

1. Con respecto a la relación entre resiliencia, funcionamiento familiar e inteligencia socioemocional en mujeres maltratadas, los hallazgos más importantes establecen correlaciones positivas y significativas entre la inteligencia emocional total y la resiliencia corroborándose la hipótesis 1 en el sentido de que las mujeres que han sido objeto de maltrato conyugal por su pareja o expareja revelan que poseen suficiente grado de control de sus emociones, lo cual les ayuda a ser resilientes.

2. Las correlaciones entre la inteligencia emocional y el funcionamiento familiar integral son significativas y negativas, al interpretar los puntajes de la escala de funcionamiento familiar, se debe de tener en cuenta que a menor puntaje las relaciones son más saludables, lo que indicaría que existe sentimientos y relaciones positivas no con la pareja o expareja, pero posiblemente con los otros miembros que integran el grupo familiar.

3. Al analizar las comparaciones considerando el grupo etario sólo se halló diferencia significativa en el componente de adaptabilidad de inteligencia emocional, denotando un mayor nivel de adaptación y también en competencia personal del factor resiliencia, las mujeres mayores cuyas edades estaban comprendidas entre los 40 a 61 años, corroborándose parcialmente la hipótesis 2.

\section{REFERENCIAS}

Almeida,S. da S., S, Omar, A., Aguiar, M., \& Carvalho,S., R. (2009). Resiliencia e inteligencia emocional en adolescentes: un estudio Brasil, Argentina y México. (Resumen). En Conselho Regional de Psicologia (Org.), Resumos de comunicaciones científicas. VI Congresso Norte Nordeste de Psicología (versión electrónica). Belém: UFPará.

Bar-On, R. (1997a). BarOn Emotional Quotient Inventory Technical Manual. Toronto: Multi-Health Systems. 
Bar-On, R. (1997b). Development of the Baron EQ-I: A measure of Emotional and Social Intelligence. Trabajo presentado en 105th Annual Convention of the American Psychological Association, Chicago.

Blázquez, M., Moreno, J.M. y García-Baamonde, M.E. (2010). Revisión teórica del maltrato psicológico en la violencia conyugal. Psicología y Salud, 20(1), 65-75. Recuperado de https://www.uv.mx/psicysalud/psicysalud-20-1/20-1/ Macarena-Blazquez-Alonso.pdf

Blázquez Alonso, M., \& Moreno Manso, J., \& García-Baamonde Sánchez, M. (2009). Inteligencia emocional como alternativa para la prevención del maltrato psicológico en la pareja. Anales de Psicología, 25 (2), 250-260.

Carolin, J. (2014). Factores de resiliencia en mujeres jefas de hogar víctimas de violencia intrafamiliar, maltrato y abuso (Tesis de Licenciatura). Universidad Rafael Landívar, Nueva Guatemala de la Asunción. Recuperada de http:// biblio3.url.edu.gt/Tesario/2014/05/42/Carolin-Jesus.pdf

Cordero, Vy Teyes, R. (mayo, 2016). Resiliencia de mujeres en situación de violencia doméstica. Omnia, 22(2), 107-118. Recuperado de http://www.redalyc.org/ pdf/737/73749821009.pdf

Epstein, N. B., Bishop, D. S. \& Levine, S. (1978). The McMaster model of family functioning. Journal of Marriage and Family Counseling, 4(4) 19-31.

Faul, F., Erdfelder, E., Buchner, A. and Lang, A.G. (2009) Statistical Power Analyses Using G*Power 3.1: Tests for Correlation and Regression Analyses. Behavior Research Methods, 41, 1149-1160. http://dx.doi.org/10.3758/BRM.41.4.1149

Faul, F., Erdfelder, E., Buchner, A. y Lang, A.G. (2014). G*Power (versión 3.1.9.2) [Software de computación]. Recuperado de http://www.gpower.hhu.de/

Fergus, S. y Zimmerman, M. A. (2005). Adolescent resilience: a framework for understanding healthy development in the face of risk. Annual Review of Public Health, 26, 399-419. Recuperado de

Gonzales, M. (2017). Violencia contra la mujer en el distrito de Santiago de Surco. Recuperado del sitio de Internet de la Universidad Ricardo Palma: http:// repositorio.urp.edu.pe/handle/urp/1080

Hernández, S. R., Fernández, C. C. y Baptista, L. P. (2014). Metodología de la Investigación. México: Mac Graw-Hill.

Lam, N., Contreras. H., Cuesta, F., Mori, E., Cordori, J., y Carrillo, N. (2008). Resiliencia y apoyo social frente a trastornos depresivos en gestantes sometidas a violencia de género. Revista peruana de epidemiología, 12(3) ,1-8. http://www. redalyc.org/html/2031/203120335005/

Congreso de la República del Perú (2015). Ley para prevenir, sancionary erradicar la violencia contra las mujeres y los integrantes del grupo familiar [Ley № 30364].

Ministerio de la Mujer y Poblaciones Vulnerables (2019). Informe estadístico. Violencia en cifras. Boletín 1 -Enero 2019. Recuperado de https://www.mimp. gob.pe/contigo/contenidos/pncontigo-articulos.php?codigo $=80$ 
Murphy, C. M. y Hoover, S. A. (1999). Measuring emotional abuse in dating relationships as a multifactorial construct. Violence and victims, 14(1), 39-53. Recuperado de https://www.ncbi.nlm.nih.gov/pubmed/10397625

Organización Mundial de la Salud (2013). Informe de la OMS destaca que la violencia contra la mujer es "un problema de salud global de proporciones epidémicas". Recuperado de http://www.who.int/mediacentre/news/ releases/2013/violence_against_women_20130620/es/

Salgado, C. (2012). Efectos del bienestar espiritual sobre la resiliencia en estudiantes universitarios de Argentina, Bolivia, Perú y República Dominicana (Tesis de Doctorado). Universidad Nacional Mayor de San Marcos, Lima. Recuperado de http://cybertesis.unmsm.edu.pe/handle/cybertesis/3293

Siegel, S. y Castellan, N. J. (1995): Estadística no Paramétrica aplicada a las ciencias de la conducta (4a ed.). México: Trillas.

Thorndike, R.C. y Hagen, E. (1989). Medicion y evaluaciòn en Psicologia y Educaciòn. Ed. Trillas. Mexico.

Ungar, M. (2010). Families as navigators and negotiators: facilitating culturally and contextually specific expressions of resilience. Family process, 49(3), 421-435. doi: 10.1111/j.1545-5300.2010.01331

Ungar, M. (2011). The social ecology of resilience. Addressing contextual and cultural ambiguity of a nascent construct. American Journal of Orthopsychiatry, 81(1), 1-17. Recuperado de http://psycnet.apa.org/record/2012-11011-001

Ugarriza, N. (2001). La evaluación de la inteligencia emocional a través del inventario de BarOn (I-CE) en una muestra de Lima Metropolitana. Persona, 4, 129-160.

Wagnild, G. y Young. H. (1993). Escala de resiliencia (ER). Recuperado de http:// es.scribd.com/doc/39013071/Escala-de-Resiliencia-de-Wagnild-y-Young

Walsh F.(2004). Resiliencia familiar. Estrategias para su fortalecimiento. Buenos Aires: Amorrortu Editores. 